\title{
Fourier-transform inelastic X-ray scattering from time- and momentum-dependent phonon-phonon correlations
}

\author{
M. Trigo ${ }^{1,2 \star}$, M. Fuchs ${ }^{1,2}$, J. Chen ${ }^{1,2}$, M. P. Jiang ${ }^{1,2}$, M. Cammarata ${ }^{3}$, S. Fahy ${ }^{4}$, D. M. Fritz ${ }^{3}$, K. Gaffney ${ }^{2}$,
} S. Ghimire ${ }^{2}$, A. Higginbotham ${ }^{5}$, S. L. Johnson ${ }^{6}$, M. E. Kozina ${ }^{2}$, J. Larsson ${ }^{7}$, H. Lemke ${ }^{3}$, A. M. Lindenberg ${ }^{1,2,8}$, G. Ndabashimiye ${ }^{2}$, F. Quirin ${ }^{9}$, K. Sokolowski-Tinten ${ }^{9}$, C. Uher ${ }^{10}$, G. Wang ${ }^{10}$, J. S. Wark ${ }^{5}$, D. Zhu ${ }^{3}$ and D. A. Reis ${ }^{1,2,11 \star}$

The macroscopic characteristics of a material are determined by its elementary excitations, which dictate the response of the system to external stimuli. The spectrum of excitations is related to fluctuations in the density-density correlations and is typically measured through frequency-domain neutron ${ }^{1}$

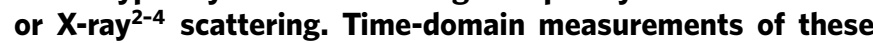
correlations could yield a more direct way to investigate the excitations of solids and their couplings both near to and far from equilibrium. Here we show that we can access large portions of the phonon dispersion of germanium by measuring the diffuse scattering from femtosecond $X$-ray freeelectron laser pulses. A femtosecond optical laser pulse slightly quenches the vibrational frequencies, producing pairs of highwavevector phonons with opposite momenta. These phonons manifest themselves as time-dependent coherences in the displacement correlations ${ }^{5}$ probed by the X-ray scattering. As the coherences are preferentially created in regions of strong electron-phonon coupling, the time-resolved approach is a natural spectroscopic tool for probing low-energy collective excitations in solids, and their microscopic interactions.

Density fluctuations in nominally periodic media reduce the intensity of the Bragg diffraction peaks and consequently increase the weak diffuse scattering between these peaks, the details of which reflect the amplitudes and spatial frequencies of the fluctuations ${ }^{6}$. The scattered intensity is determined by the dynamic structure factor $S(\mathbf{Q}, \omega)$ at momentum $\mathbf{Q}$ and frequency $\omega$, which is proportional to the Fourier transform of the correlation function of the density-density fluctuations. For phonons, these correlations are $\left\langle u_{\mathbf{q}}(0) u_{-\mathbf{q}}(t)\right\rangle$, where $u_{\mathbf{q}}$ is the phonon amplitude at reduced wavevector $\mathbf{q}=\mathbf{Q}-\mathbf{K}_{\mathbf{Q}}$ and $\mathbf{K}_{\mathbf{Q}}$ is the closest reciprocal lattice vector to $\mathbf{Q}$, and in this context the expectation value is a thermal average ${ }^{7}$. In typical $\mathrm{X}$-ray or neutron scattering experiments the measured diffuse scattering is proportional to the equal-time correlations $\left\langle u_{\mathbf{q}}(0) u_{-\mathbf{q}}(0)\right\rangle$ (refs $3,7,8$ ); whereas dynamic information is obtained by analysing the energy and momentum of the inelastically scattered photons from a highly monochromatic beam. As we demonstrate here in a single crystal of the prototypical semiconductor germanium, a femtosecond laser pulse generates temporal coherences in the equal-time correlation functions $g(\tau)=\left\langle u_{\mathbf{q}} u_{-\mathbf{q}}\right\rangle$ parameterized by the pump-probe delay $\tau$ between the optical pulse and the X-ray probe. As the X-ray pulse from the free-electron laser (FEL) is short compared with the vibrational motion, we assume that the scattering is effectively instantaneous. Under this approximation we measure $g(\tau)$ stroboscopically, which unlike in the thermal case has an oscillatory contribution from a two-phonon squeezed state generated by the laser pulse, as well as a contribution from incoherent changes in populations ${ }^{9}$. In this paper we focus on the oscillatory component, which yields large portions of the phonon dispersion directly from the measurement without any particular model of the interatomic forces.

Consider a sudden change in the harmonic potential driven by excitation of electron-hole pairs by the laser pulse, which for tetrahedrally bonded semiconductors is expected to primarily soften the transverse acoustic modes ${ }^{10-13}$. The evolution of a harmonic oscillator after a sudden quench of the frequency has been studied in the context of vacuum squeezing, as shown for photons ${ }^{14}$ and phonons ${ }^{15,16}$. This effect is formally equivalent to the dynamical Casimir effect ${ }^{17}$ and its acoustic analogue in which a sudden quench of the sound velocity was shown to produce correlated pairs of phonons ${ }^{18}$, and is analogous to (spontaneous) parametric downconversion. Although our experiment was performed at room temperature, and the results are due to thermal rather than vacuum squeezing, we consider the zero temperature case for simplicity.

For oscillators with frequencies $\Omega_{\mathbf{q}}$ and mass $m$ in the ground state, a sudden change in the frequency $\Omega_{\mathbf{q}} \rightarrow \Omega_{\mathbf{q}}^{\prime}$ at $\tau=0$ leaves each mode in a state where the variance in the displacement evolves according to ${ }^{19}$

$$
\left\langle u_{\mathbf{q}} u_{-\mathbf{q}}\right\rangle=\frac{1}{4 m \Omega_{\mathbf{q}}}\left[\left(1+\beta_{\mathbf{q}}^{2}\right)+\left(1-\beta_{\mathbf{q}}^{2}\right) \cos \left(2 \Omega_{\mathbf{q}}^{\prime} \tau\right)\right]
$$

\footnotetext{
${ }^{1}$ Stanford Institute for Materials and Energy Sciences, SLAC National Accelerator Laboratory, Menlo Park, California 94025, USA, ${ }^{2}$ Stanford PULSE Institute, SLAC National Accelerator Laboratory, Menlo Park, California 94025, USA, ${ }^{3}$ Linac Coherent Light Source, SLAC National Accelerator Laboratory, Menlo Park, California 94025, USA, ${ }^{4}$ Tyndall National Institute and Department of Physics, University College, Cork, Ireland, ${ }^{5}$ Department of Physics, Clarendon Laboratory, University of Oxford, Parks Road, Oxford OX1 3PU, UK, ${ }^{6}$ Physics Department, ETH Zurich, 8093 Zurich, Switzerland, ${ }^{7}$ Department of Physics, Lund University, S-22100 Lund, Sweden, ${ }^{8}$ Department of Materials Science and Engineering, Stanford University, Stanford, California 94305 , USA, ${ }^{9}$ Faculty of Physics and Center for Nanointegration Duisburg-Essen (CENIDE), University of Duisburg-Essen, 47048, Duisburg, Germany, ${ }^{10}$ Department of Physics, University of Michigan, Ann Arbor, Michigan 48109, USA, ${ }^{11}$ Departments of Photon Science and Applied Physics, Stanford University, Stanford, California 94305, USA. *e-mail:mtrigo@slac.stanford.edu; dreis@slac.stanford.edu
} 

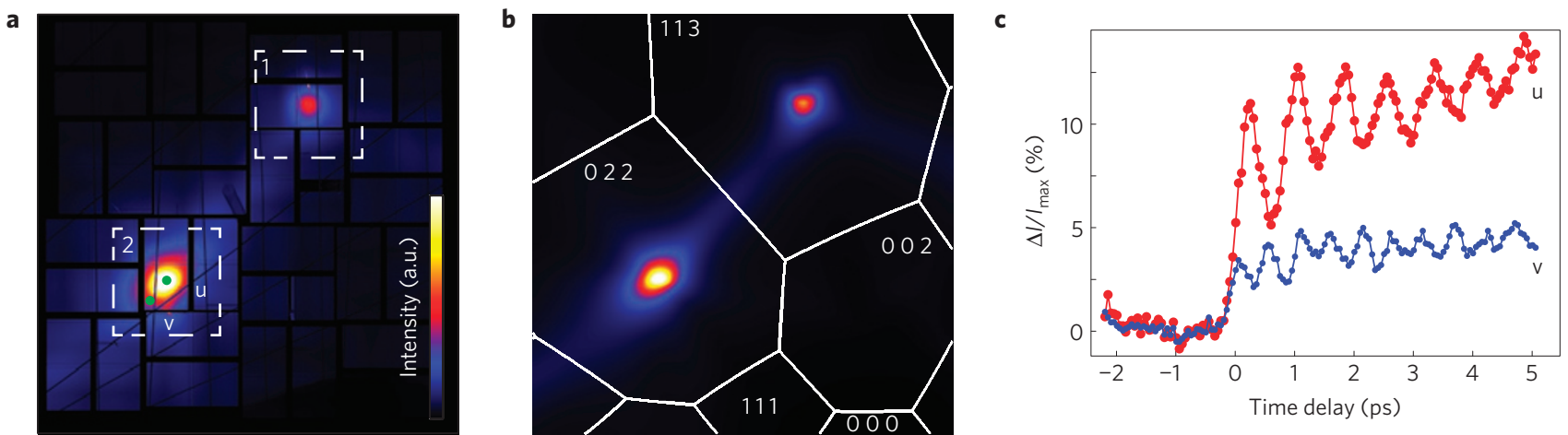

Figure 1 | Femtosecond X-ray diffuse scattering. a, Static thermal diffuse scattering from (001) Ge in grazing incidence from 10 keV X-ray photons at the LCLS. Dashed squares are the q-space regions shown in Fig. 3. b. Calculated equilibrium pattern using a Born model of the forces. White lines indicate the boundaries of the Brillouin zones. Miller indices are also indicated. $\mathbf{c}$, Representative traces of the normalized change in scattering $\Delta /(t) / I_{\max }$ induced by the optical laser as a function of (optical) pump-(X-ray) probe delay.

where $\beta_{\mathrm{q}}=\Omega_{\mathbf{q}} / \Omega_{\mathrm{q}}^{\prime}>1$ for a sudden softening. This expression describes the evolution of correlated pairs of phonons at $\mathbf{q}$ and $-\mathbf{q}$ (ref. 20). Accordingly, the diffuse scattering intensity oscillates at $2 \Omega^{\prime}$, with an amplitude proportional to $1-\beta_{\mathbf{q}}^{2}$. In the limit of low-density excitation, the frequencies will approximate the equilibrium values, and thus the Fourier transform of the oscillatory component should give the phonon dispersion. At finite temperatures, equation (1) contains an additional thermal factor ${ }^{20}$. We emphasize that the excitations described above have $\langle u(\tau)\rangle=0$ and thus are squeezed states and not coherent states. This is expected because the small wavevector of the visible light cannot impart enough momentum to the lattice to generate coherent phonons at large $\mathbf{q}$ and thus can generate only pairs of phonons with equal and opposite momenta ${ }^{15}$. In our case, the softening is expected to occur for all $\mathbf{q}$ and be particularly strong at the Brillouin zone boundary ${ }^{12,13}$.

The experiments were performed at the Linac Coherent Light Source (LCLS) X-ray FEL using nominally $50 \mathrm{fs,} 1.55 \mathrm{eV}$ pump pulses and $50 \mathrm{fs}, 10 \mathrm{keV}$ X-ray probe pulses (see Supplementary Information for details). In Fig. 1a we plot a portion of the equilibrium X-ray diffuse scattering, without laser excitation, from a single crystal of germanium at grazing incidence, captured with an area detector. The signal is proportional to the energy-integrated dynamic structure factor, $S(\mathbf{Q})$, as the detector lacks energy resolution. The bright areas correspond to regions of reciprocal space with low-frequency acoustic phonons that contribute strongly to the equilibrium diffuse scattering ${ }^{9,21}$. Figure $1 \mathrm{~b}$ shows the simulated thermal diffuse scattering from a Born-von Karman model of the forces including interactions up to six nearest neighbours ${ }^{22-24}$ (see Supplementary Information for additional details). This simple model describes well the phonon dispersion including the flattening of the transverse acoustic branches ${ }^{25}$. The calculated pattern matches the measured diffuse scattering extremely well. The white solid contours in Fig. 1b represent the boundaries of the Brillouin zones accessible in this geometry, and we have also indicated the respective Miller indices. Figure 1c shows the evolution of the change in normalized diffuse scattering intensity $\Delta I(\tau) / I_{\max } \propto g(\tau)$, induced by photoexcitation with a $50 \mathrm{fs}$ infrared laser pulse centred at $800 \mathrm{~nm}$. The two curves show the time traces for the two points labelled $u$ and $v$ in Fig. 1a, normalized by the maximum of the laser-off image. Photoexcitation induces an overall step-like increase in the scattering whose magnitude depends on momentum, and oscillations at frequencies in the range of $1-3.5 \mathrm{THz}$. In our case, $\left|1-\beta_{\mathbf{q}}^{2}\right| \approx 0.05$ and thus $\Omega_{\mathbf{q}} / \Omega_{\mathbf{q}}^{\prime} \approx 1.025$, such that the expected frequency difference is close to the resolution limit of $\sim 0.05 \mathrm{THz}$ given by the finite time window in these data. Consistent with bond softening the mean square displacements (and thus the scattering) increases during the first quarter cycle. The sharpness of the initial step and the highest frequency observed $\sim 3.5 \mathrm{THz}$ were limited by the timing jitter in the pump-probe delay $\sim 250$ fs (ref. 26).

For better sensitivity to the oscillatory signal we filtered the slowly varying background from the raw data. Figure 2 a shows representative frames of the obtained oscillatory component in the (022) Brillouin zone. The data for zone (113) show qualitatively similar results but for a different slice of reciprocal space. The red (blue) regions in this figure represent an increase (decrease) in the intensity relative to the subtracted average. The fringes in $\mathbf{q}$ space seen here originate from phonons with different frequencies across reciprocal space, which have phase coherence due to the sudden frequency softening. The traces in Fig. $2 b$ show some of these oscillations for a few wavevectors along the $\mathrm{u}-\mathrm{v}$ segment in Fig. 1a. Movies of the raw and filtered data are available in the Supplementary Information.

In Fig. 3, we show an expanded view at selected frequencies of the Fourier transform along the time axis of the oscillatory component in Fig. 2 (top and bottom rows represent regions in squares 1 and 2 in Fig. 1a, respectively). The value of each pixel is the magnitude of the Fourier transform at a given frequency of traces such as those shown in Fig. 2b. The bright loops appear at locations in momentum space where the intensity oscillates at the same frequency. These contours (Fig. 3) represent constant-frequency cuts of the phonon dispersion relation as depicted schematically in Fig. 4a. The differences between the data in the two regions in Fig. 3 are due to the different reciprocal space areas sampled by the two Brillouin zones and thus originate from different phonon modes. The data in Fig. 3 show two bands, seen more clearly in the bottom row plots, which correspond to the two transverse acoustic branches, with pinch points where the bands are degenerate along high-symmetry directions. Their intensity depends on the amplitude of the coherent mean squared displacements, as well as their projection along $\mathbf{Q}$.

Finally, Fig. 4c,d shows the extracted dispersion relation along the directions indicated by the dashed lines in Fig. $4 \mathrm{~b}$. Here the phonon frequency is $\Omega \approx \Omega^{\prime}=\omega / 2$ according to equation (1). Again we stress that we have not relied on any model of the interatomic forces to extract the phonon frequencies. For comparison, the white lines in Fig. 4c,d show the calculated equilibrium dispersion. Note that within our experimental sensitivity we pick out only the transverse acoustic phonon branches and not the longitudinal acoustic branch. (At present the time resolution is not high enough to resolve either optical phonon branch.) This is to be expected as the excitation of carriers reduces the strength of the covalent bonds that give rise to the shear stability in the tetrahedrally 

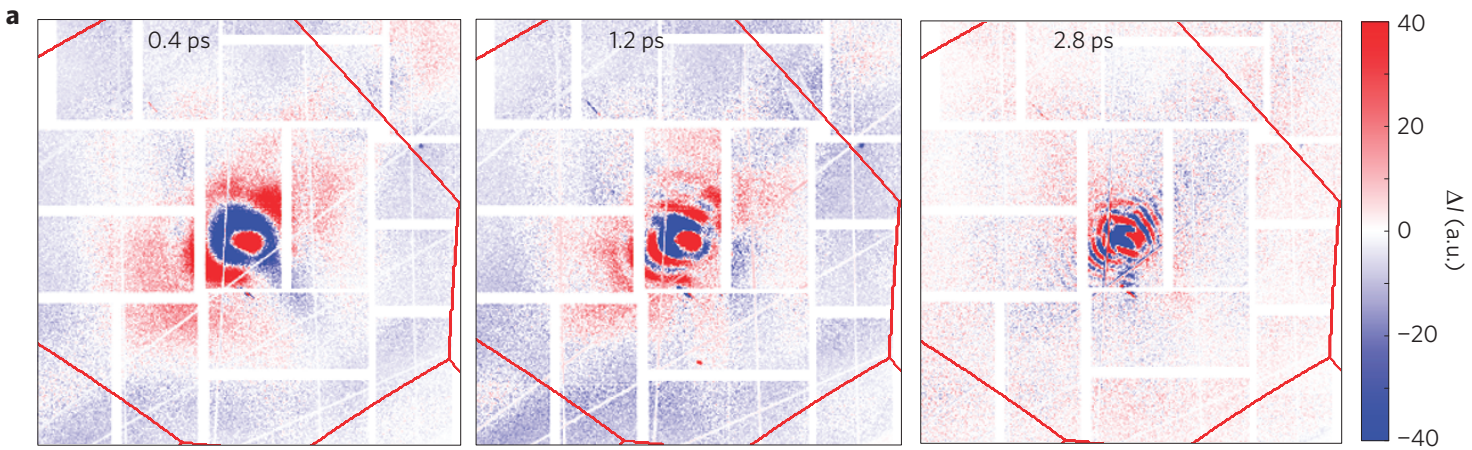

b

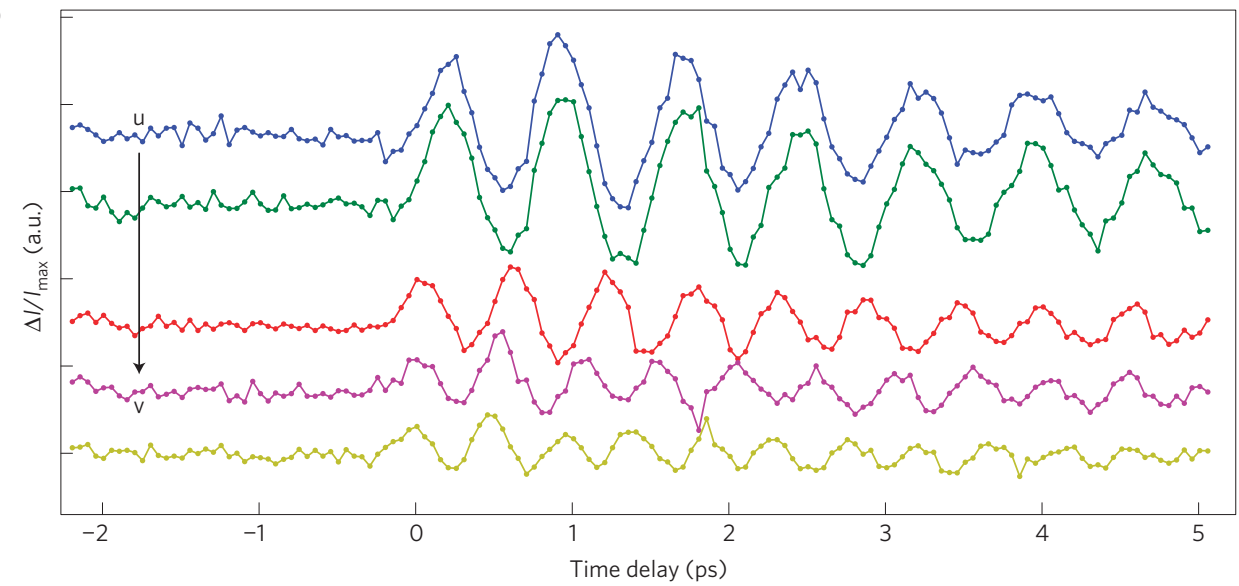

Figure 2 | Coherence in the density-density correlations. a, Representative frames of the oscillatory component of $\Delta l / I_{\max }$ after background subtraction. b, Time dependence of the subtracted data at a few reduced wavevector locations between $u=(-0.1,0.00,-0.08)$ and $v=(-0.03,0.15,-0.27)(r .1$.u. $)$ in Fig. 1a. These curves have been displaced vertically for clarity.
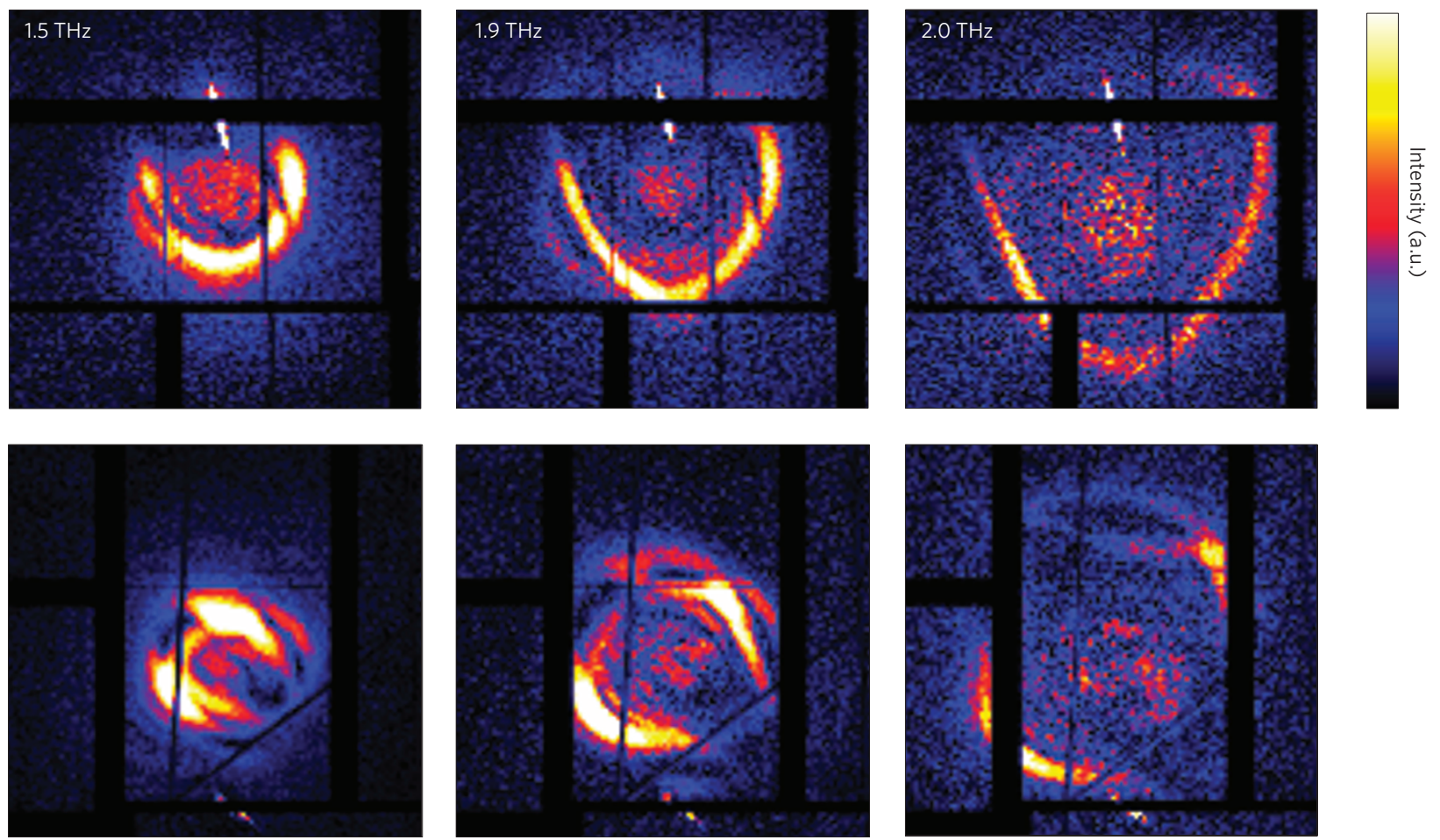

Figure 3 | Constant-frequency phonon momentum distribution. Magnitude of the time Fourier transform at representative frequencies of the background-subtracted data. The colour bar indicates relative units on a linear scale. Top and bottom panels, zoomed view of the region of $\mathbf{q}$-space labelled 1 and 2 in Fig. 1, respectively. 


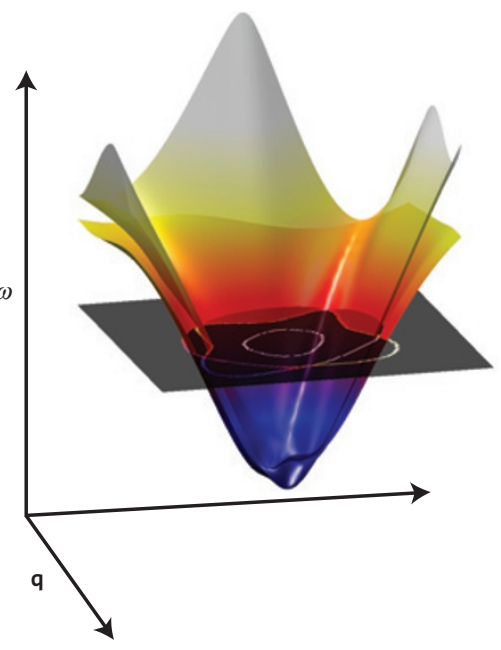

b

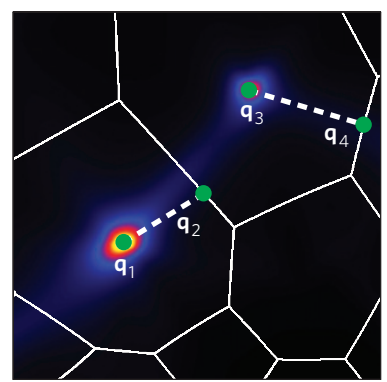

C

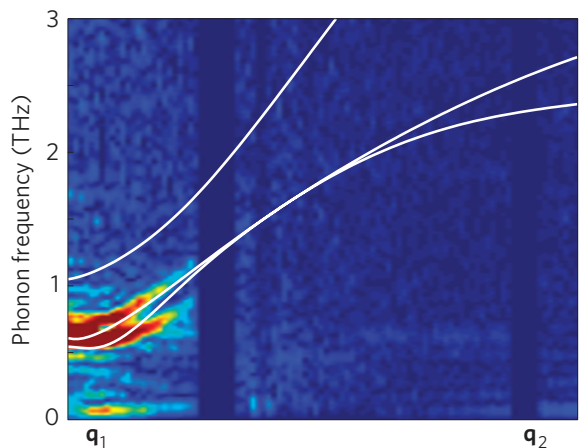

d

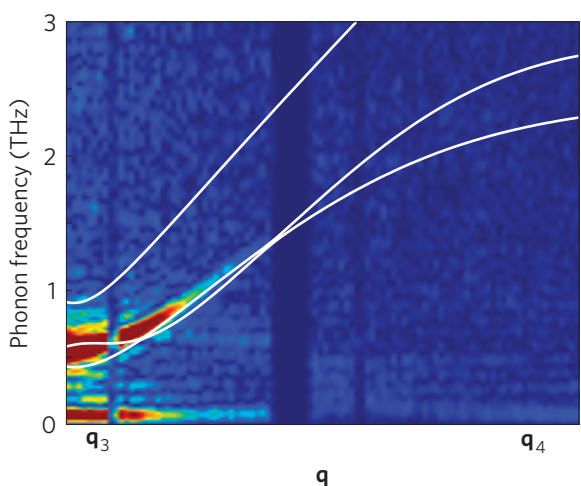

Figure 4 | Extracted dispersion relation in selected directions. a, Schematic of the constant-frequency cuts of the acoustic dispersion relation that yield the data in Fig. 3. The surfaces represent the two transverse-acoustic branches and the plane represents a constant-frequency cut at $2 \omega=2.5 \mathrm{THz}$. b-d, Acoustic dispersion along the sections shown with dashed lines on the calculated intensity $(\mathbf{b})$ where $\mathbf{q}_{1}=(-0.1,0,-0.07)$, $\mathbf{q}_{2}=(-0.33,-0.75,0.37),(\mathbf{c}), \mathbf{q}_{3}=(0.13,-0.04,0.05)$ and $\mathbf{q}_{4}=(-0.09,-0.98,-0.08)(\mathrm{r} . \mathrm{l} . \mathrm{u})$ (d). White lines in $\mathbf{c}, \mathbf{d}$ represent the calculated acoustic dispersion.

bonded semiconductors. Otherwise, the discrepancies are small and could be due to systematic errors in determining the sample orientation or the forces as much as changes in the excited-state forces. The curvature of the branches is due to our particular geometry, which results in a non-planar section of reciprocal space. The flat spectral components at lower frequencies are probably due to fluctuations of the FEL that were not removed by our background subtraction. The sample was oriented far from the zone-centre $(\mathbf{q}=0)$ to avoid strong Bragg reflections on the detector, particularly given the large wavelength fluctuations of the FEL.

We note that the present experiment was limited by the FEL and laser parameters as well as detector performance as available shortly after hard X-ray operations of the LCLS commenced. Recently, self-seeded operation of the LCLS has been demonstrated ${ }^{27}$. This provides better X-ray pulse stability yielding better momentum resolution, lower noise, and the narrow bandwidth will allow sampling closer to $\mathbf{q}=0$. In addition, a new single-shot timing diagnostic has been reported that mitigates the loss in temporal resolution due to timing jitter between the optical and X-ray lasers $^{28}$. This enables the observation of faster oscillations and thus higher-frequency excitations limited by the pump and probe pulse duration. We further note that the FEL can operate with pulses down to a few femtoseconds long, and optical lasers with pulse durations in the few tens of femtosecond range are readily available. These improvements will allow access to high-frequency optical phonons modes in the $>10 \mathrm{THz}$ range such as those in many complex oxide materials.

The induced temporal coherences in the density-density correlations observed here are a consequence of a sudden change in the interatomic potential. These coherences span the entire Brillouin zone but will be favoured in regions where the resultant (real or virtual) charge-density couples strongly to the phonons. For example, it will be particularly strong in regions of enhanced electron-phonon coupling and could find broad use in the study of the coupled degrees of freedom in complex materials. We further stress that, far from equilibrium the pump-probe approach gives unique access to the phonon excitations and their interactions in the short-lived transient state.

\section{Received 14 March 2013; accepted 11 September 2013;} published online 27 October 2013

\section{References}

1. Brockhouse, B. N. \& Stewart, A. T. Scattering of neutrons by phonons in an aluminum single crystal. Phys. Rev. 100, 756-757 (1955).

2. Rueff, J-P. \& Shukla, A. Inelastic x-ray scattering by electronic excitations under high pressure. Rev. Mod. Phys. 82, 847-896 (2010).

3. Krisch, M. \& Sette, F. in Light Scattering in Solid IX Vol. 108 (eds Cardona, M. \& Merlin, R.) 317-370 (Topics in Applied Physics, Springer, 2007).

4. Abbamonte, P., Finkelstein, K. D., Collins, M. D. \& Gruner, S. M. Imaging density disturbances in water with a 41.3-attosecond time resolution. Phys. Rev. Lett. 92, 237401 (2004).

5. Glauber, R. J. Time-dependent displacement correlations and inelastic scattering by crystals. Phys. Rev. 98, 1692-1698 (1955).

6. Warren, B. E. X-Ray Diffraction (Dover, 1969).

7. Sinha, S. K. Theory of inelastic $\mathrm{x}$-ray scattering from condensed matter. J. Phys. Condens. Matter 13, 7511-7523 (2001).

8. Van Hove, L. Correlations in space and time and born approximation scattering in systems of interacting particles. Phys. Rev. 95, 249-262 (1954).

9. Trigo, M. et al. Imaging nonequilibrium atomic vibrations with $\mathrm{x}$-ray diffuse scattering. Phys. Rev. B 82, 235205 (2010). 
10. Biswas, R. \& Ambegaokar, V. Phonon spectrum of a model of electronically excited silicon. Phys. Rev. B 26, 1980-1988 (1982).

11. Stampfli, P. \& Bennemann, K. H. Theory for the instability of the diamond structure of $\mathrm{Si}, \mathrm{Ge}$, and $\mathrm{C}$ induced by a dense electron-hole plasma. Phys. Rev. B 42, 7163-7173 (1990).

12. Zijlstra, E. S., Walkenhorst, J. \& Garcia, M. E. Anharmonic noninertial lattice dynamics during ultrafast nonthermal melting of InSb. Phys. Rev. Lett. 101, 135701 (2008).

13. Hillyard, P. B., Reis, D. A. \& Gaffney, K. J. Carrier-induced disordering dynamics in InSb studied with density functional perturbation theory. Phys. Rev. B 77, 195213 (2008).

14. Slusher, R. E., Hollberg, L. W., Yurke, B., Mertz, J. C. \& Valley, J. F. Observation of squeezed states generated by four-wave mixing in an optical cavity. Phys. Rev. Lett. 55, 2409-2412 (1985).

15. Garrett, G. A., Rojo, A. G., Sood, A. K., Whitaker, J. F. \& Merlin, R. Vacuum squeezing of solids: Macroscopic quantum states driven by light pulses. Science 275, 1638-1640 (1997)

16. Johnson, S. L. et al. Directly observing squeezed phonon states with femtosecond x-ray diffraction. Phys. Rev. Lett. 102, 175503 (2009).

17. Nation, P. D., Johansson, J. R., Blencowe, M. P. \& Nori, F. Stimulating uncertainty: Amplifying the quantum vacuum with superconducting circuits. Rev. Mod. Phys. 84, 1-24 (2012).

18. Jaskula, J-C. et al. Acoustic analog to the dynamical Casimir effect in a Bose-Einstein condensate. Phys. Rev. Lett. 109, 220401 (2012).

19. Kiss, T., Janszky, J. \& Adam, P. Time evolution of harmonic oscillators with time-dependent parameters: A step-function approximation. Phys. Rev. A 49, 4935-4942 (1994).

20. Carusotto, I., Balbinot, R., Fabbri, A. \& Recati, A. Density correlations and analog dynamical Casimir emission of Bogoliubov phonons in modulated atomic Bose-Einstein condensates. Eur. Phys. J. D 56, 391-404 (2010).

21. Ruqing, X. \& Chiang, T. C. Determination of phonon dispersion relations by $\mathrm{X}$-ray thermal diffuse scattering. Z. Kristallogr. 220, 1009-1016 (2005).

22. Holt, M. et al. Determination of phonon dispersions from $\mathrm{x}$-ray transmission scattering: The example of silicon. Phys. Rev. Lett. 83, 3317-3319 (1999).

23. Herman, Frank Lattice vibrational spectrum of germanium. J. Phys. Chem. Solids 8, 405-418 (1959).

24. Patel, C., Sherman, W. F. \& Wilkinson, G. R. Reinvestigation of the lattice dynamics of diamond on the basis of a born-von kármán model. J. Phys. C. Solid State Phys. 17, 6063-6069 (1984).

25. Jian, W., Kaiming, Z. \& Xide, X. Reinvestigation of the TA modes in Ge and Si in Born-von Karman model. Solid State Commun. 86, 731-734 (1993).
26. Glownia, J. M. et al. Time-resolved pump-probe experiments at the LCLS. Opt. Express 18, 17620-17630 (2010).

27. Amann, J. et al. Demonstration of self-seeding in a hard-x-ray free-electron laser. Nature Photon. 6, 693-698 (2012).

28. Harmand, M. et al. Achieving few-femtosecond time-sorting at hard x-ray free-electron lasers. Nature Photon. 7, 215-218 (2013).

\section{Acknowledgements}

The authors thank A. Barty, M. Bionta, J. Defever, S. Edstrom, C. Kenney, T. Huber, S. Nelson and K. Ramsey for their experimental assistance. This work was primarily supported by the US Department of Energy (DOE), Office of Basic Energy Sciences (BES) through the Division of Materials Sciences and Engineering under contract DE-AC02-76SF00515. Measurements were carried out at the LCLS at SLAC National Accelerator Laboratory. LCLS is an Office of Science User Facility operated for DOE Office of Science by Stanford University. M.E.K. was supported by the DOE Office of Science Graduate Fellowship Program. G.N. and S.G. were supported by the AMOS program within the Chemical Sciences, Geosciences, and Biosciences Division, DOE, BES. M.F. acknowledges financial support from the Volkswagen Foundation. F.Q. and K.S-T. acknowledge support by the German Research Council (DFG) through the Collaborative Research Center 616 'Energy Dissipation at Surfaces'. J.L. was supported by the Swedish Science Council (VR) A.H. was supported by AWE. J.S.W. is grateful for support from the UK EPSRC under grant no. EP/H035877/1.

\section{Author contributions}

D.A.R. and M.T. conceived the experiment and the framework for the data interpretation, M.T. and M.F. analysed the data, K.G., S.F. and J.S.W. contributed to data interpretation, C.U. and G.W. prepared Bi samples for the precise timing overlap between the X-ray and optical pulses, and M.T. and D.A.R. wrote the manuscript with input from all other authors. The experiment was carried out by M.T., M.F., J.C., M.P.J., M.C., D.M.F., K.G, S.G., A.H., S.L.J., M.E.K., J.L., H.L., A.M.L., G.N., F.Q., K.S-T., D.Z. and D.A.R. The X-ray pump-probe instrument was operated by M.C. D.M.F., H.L. and D.Z

\section{Additional information}

Supplementary information is available in the online version of the paper. Reprints and permissions information is available online at www.nature.com/reprints. Correspondence and requests for materials should be addressed to M.T. or D.A.R.

\section{Competing financial interests}

The authors declare no competing financial interests. 